\title{
Topological Hall effect in thin films of $\mathrm{Mn}_{1.5} \mathrm{PtSn}$
}

\author{
Peter Swekis, ${ }^{1,2}$ Anastasios Markou, ${ }^{1, *}$ Dominik Kriegner, ${ }^{1}$ Jacob Gayles, ${ }^{1}$ Richard Schlitz, ${ }^{2,3}$ Walter Schnelle, \\ Sebastian T. B. Goennenwein, ${ }^{2,3}$ and Claudia Felser ${ }^{1, \dagger}$ \\ ${ }^{1}$ Max-Planck Institute for Chemical Physics of Solids, 01187 Dresden, Germany \\ ${ }^{2}$ Institut für Festkörper- und Materialphysik, Technische Universität Dresden, 01062 Dresden, Germany \\ ${ }^{3}$ Center for Transport and Devices of Emergent Materials, Technische Universität Dresden, 01062 Dresden, Germany
}

(Received 23 October 2018; published 2 January 2019)

\begin{abstract}
Spin chirality in metallic materials with noncoplanar magnetic order can give rise to a Berry phase induced topological Hall effect. Here, we report the observation of a large topological Hall effect in high-quality films of $\mathrm{Mn}_{1.5} \mathrm{PtSn}$ that were grown by means of magnetron sputtering on $\mathrm{MgO}(001)$. The topological Hall resistivity is present up to $\mu_{0} H \approx 4 \mathrm{~T}$ below the spin reorientation transition temperature, $T_{s}=185 \mathrm{~K}$. We find that the maximum topological Hall resistivity is of comparable magnitude as the anomalous Hall resistivity. Owing to the size, the topological Hall effect is directly evident prior to the customarily performed subtraction of magnetometry data. Further, we underline the robustness of the topological Hall effect in $\mathrm{Mn}_{2-x} \mathrm{PtSn}$ by extracting the effect for multiple stoichiometries $(x=0.5,0.25,0.1)$ and film thicknesses $(t=104,52,35 \mathrm{~nm})$ with maximum topological Hall resistivities between 0.76 and $1.55 \mu \Omega \mathrm{cm}$ at $150 \mathrm{~K}$.
\end{abstract}

DOI: 10.1103/PhysRevMaterials.3.013001

\section{INTRODUCTION}

Topological magnetic structures have become of great interest recently, attributed to the emergent transport phenomena associated with the magnetic texture [1]. One of these phenomena is the transverse Hall current, that arises from the interplay of magnetic order and intrinsic band structure or scattering [2]. Experimentally, the measured Hall resistivity can be separated into the ordinary Hall effect (OHE) [3] dependent on the external field $(H)$ and the anomalous Hall effect (AHE) which scales with the saturation magnetization $\left(M_{s}\right)$. The modern understanding of the AHE ascribes the effect to scattering mechanisms [4-6] and the intrinsic momentum space Berry curvature [7]. However, recently an additional Hall-type contribution was proposed that scales neither with the magnetization $(M)$ nor with the externally applied field, termed the topological Hall effect (THE) [8,9]. This THE has been proposed to originate from a finite scalar spin chirality [10], skyrmions [8], and Weyl points [11]. The prior two are connected to the magnetic texture and the latter is connected to the momentum space dispersion. Here, we focus on the magnetic texture induced THE which has become of great interest in Heusler compounds due to their tunability $[12,13]$.

There are two limiting cases for the stabilization of magnetic textures: the scalar spin chirality and the skyrmionic

\footnotetext{
*Anastasios.Markou@cpfs.mpg.de

${ }^{\dagger}$ Claudia.Felser@cpfs.mpg.de
}

Published by the American Physical Society under the terms of the Creative Commons Attribution 4.0 International license. Further distribution of this work must maintain attribution to the author(s) and the published article's title, journal citation, and DOI. lattice [14], which originate from a competition of exchange, e.g., Heisenberg and Dzyaloshinki-Moriya interaction $[15,16]$, with anisotropy and external fields. In the limit of discrete spins, there is a finite scalar spin chirality $S_{i} \cdot\left(S_{j} \times S_{k}\right)$ caused by three noncoplanar spins that subtend a finite cone angle and give rise to the momentum space dependent THE $[10,17]$. In the adiabatic limit, the spin chirality is taken to be continuous as the integer winding of the real space Berry curvature $[8,9,18]$. As electrons couple to such spin textures, they acquire a finite Berry phase acting as a magnetic field. This in turn results in an additional contribution to the Hall effect [19].

The THE has been observed in a variety of materials including the B20 compounds [18,20,21], perovskites [22,23], and Heusler compounds [24-26]. The Heusler compounds are of particular interest, owing to the recent discovery of antiskyrmions in $\mathrm{Mn}_{1.4} \mathrm{Pt}_{0.9} \mathrm{Pd}_{0.1} \mathrm{Sn}$, a new type of topological texture due to the $D_{2 d}$ symmetry [27]. The ferrimagnetic $\mathrm{Mn}_{2} Y Z$ ( $Y$ being a transition metal and $Z$ a main-group element) inverse Heusler compounds that crystallize in a noncentrosymmetric structure with $D_{2 d}$ symmetry are promising candidates to realize such spin textures through competing interactions of the magnetic sublattices and magnetocrystalline anisotropy caused by tetragonal distortion [28]. In thin films the presence of geometric constraints can additionally stabilize the desired spin textures in a wider field and temperature range [29]. Recently, the THE was observed in single-crystal thin films of $\mathrm{Mn}_{2} \mathrm{RhSn}$ [24] as well as in bulk $\mathrm{Mn}_{2} \mathrm{PtSn}$ [25] below a spin reorientation transition temperature $\left(T_{s}\right)$ [28], and in polycrystalline $\mathrm{Mn}_{2} \mathrm{PtSn}$ films [26] for all temperatures below the Curie temperature. Conversely, the work of Jin et al. shows no topological Hall signal or $T_{s}$ in epitaxially grown films of $\mathrm{Mn}_{2} \mathrm{PtSn}$ [30].

In this Rapid Communication, we focus on $\mathrm{Mn}_{1.5} \mathrm{PtSn}$ thin films, with the closest stoichiometry relation to the 
antiskyrmion compound $\mathrm{Mn}_{1.4} \mathrm{Pt}_{0.9} \mathrm{Pd}_{0.1} \mathrm{Sn}$. We demonstrate the presence of a THE below a spin reorientation transition temperature $T_{s}$ and up to high fields, evident prior to the customarily performed subtraction of magnetometry data. Further, we point out the robustness of the THE in $\mathrm{Mn}_{2-x} \mathrm{PtSn}$ by comparing different compositions and film thicknesses, as well as previously reported results on $\mathrm{Mn}_{2} \mathrm{PtSn}$ films.

\section{EXPERIMENTAL DETAILS}

High-quality $\mathrm{Mn}_{2-x} \mathrm{PtSn}$ films were grown on singlecrystal $\mathrm{MgO}(001)$ substrates in a BESTEC UHV magnetron sputtering system. $\mathrm{Mn}, \mathrm{Pt}$, and $\mathrm{Sn}$ were deposited from $2^{\prime \prime}$ targets using dc magnetron cosputtering. The stoichiometry was controlled by adjusting the power of the magnetrons. The deposition was performed in confocal geometry with a target to substrate distance of $200 \mathrm{~mm}$. Prior to deposition, the chamber was evacuated to a base pressure below $2 \times 10^{-8}$ mbar, while during deposition a process gas pressure of $3 \times$ $10^{-3}$ mbar (Ar, $15 \mathrm{sccm}$ ) was maintained. The films were deposited at $350^{\circ} \mathrm{C}$ and postannealed for $30 \mathrm{~min}$ at the same temperature in order to improve the chemical ordering. The annealed films were capped at room temperature with $3 \mathrm{~nm}$ $\mathrm{Al}$, in order to prevent oxidization.

The film compositions were confirmed using energydispersive $\mathrm{x}$-ray microscopy, with an experimental uncertainty of about 2 at. \%. The film surface topography was analyzed by atomic force microscopy (AFM) on an Asylum Research MFP-3D Origin by Oxford Instruments. Structural characterization was carried out using $\mathrm{x}$-ray diffractometry with $\mathrm{Cu} K \alpha 1$ radiation $(\lambda=1.5406 \AA)$ on a PANalytical X'Pert PRO system. The film thickness $(t)$ was determined by $\mathrm{X}$-ray reflectivity (XRR) measurements.

Magnetization measurements were performed on a vibrating sample magnetometer (MPMS 3; Quantum Design). In order to infer the magnetization of the films, we subtracted the diamagnetic substrate contribution as well as a lowtemperature paramagnetic contribution from the raw data. Here, the paramagnetic contribution can be attributed to impurities in the $\mathrm{MgO}$ substrate. The diamagnetic susceptibility $\left(\chi=-19.066 \times 10^{-6}\right)$ of $\mathrm{MgO}$ was determined from reference measurements. The paramagnetic contribution was fitted and subtracted from the raw data using the Brillouin function.

Four-probe and five-probe measurements were performed to obtain the resistivity along the longitudinal direction and the Hall resistivity, respectively. Therefore, an in-plane current, $I_{x}=50 \mu \mathrm{A}$, was applied along a film stripe with a width of $W=1.29 \mathrm{~mm}$ ( $y$ direction). Voltages were recorded simultaneously along the current direction $\left(V_{x}\right)$, with a lead distance of $L=7.4 \mathrm{~mm}$ ( $x$ direction, corresponding to $\mathrm{MgO}[100]$ ), as well as perpendicular to the current direction $\left(V_{y}\right)$, with a lead distance of $w=0.77 \mathrm{~mm}$ ( $y$ direction, corresponding to $\mathrm{MgO}[010])$. The magnetic field was applied along the out-of-plane $(z)$ direction $(\mathrm{MgO}[001])$. In order to obtain a clean resistivity, $\rho_{x x}$, the raw resistivity, $\rho_{x x}^{\text {raw }}=V_{x} W t /\left(L I_{x}\right)$, was symmetrized by averaging $\rho_{x x}^{\text {raw }}$ at positive and negative fields with respect to the field sweep directions. To obtain a clean Hall resistivity, $\rho_{x y}$, the raw Hall resistivity, $\rho_{x y}^{\text {raw }}=V_{y} W t /\left(w I_{x}\right)$, was antisymmetrized by averaging the difference of $\rho_{x y}^{\text {raw }}$ at positive and negative fields with respect to the field sweep directions.

\section{RESULTS AND DISCUSSION}

\section{A. Structural characterization}

In the following, we discuss the properties of a $\mathrm{Mn}_{1.5} \mathrm{PtSn}$ thin film in detail, since it has the closest stoichiometry relation to the antiskyrmion compound $\mathrm{Mn}_{1.4} \mathrm{Pt}_{0.9} \mathrm{Pd}_{0.1} \mathrm{Sn}$. In Fig. 1(a) we show the XRR, together with AFM analysis, confirming the smoothness of the film with a rms roughness of $0.3 \mathrm{~nm}$ in the obtained $5 \mu \mathrm{m} \times 5 \mu \mathrm{m}$ scan. The Kiessig fringes, reaching beyond the measurement range, are further evidence of a high-quality surface as well as a high-quality substrate to film interface. A thickness of $104.7 \mathrm{~nm}$ and a roughness of less than $0.5 \mathrm{~nm}$ is inferred from XRR fitting.

Furthermore, we use X-ray diffraction radial scans $(\omega-$ $2 \theta$ ) as shown in Figs. 1(b) and S1 (see the Supplemental Material [31]) to determine the crystal structure of our film. The symmetric radial scans in Fig. 1(b) confirm epitaxial growth since only the $(h 00)$ series of Bragg peaks, attributed to the $\mathrm{Mn}_{1.5} \mathrm{PtSn}$ film, can be observed. The full width at half maximum of the (400) out-of-plane rocking curve $\left(1.147^{\circ}\right)$ verifies high crystallinity. Additionally, more than ten asymmetric Bragg peaks (Fig. S1) can be indexed using a unit cell similar to bulk $\mathrm{Mn}_{1.4} \mathrm{PtSn}$ [27]. Analogous to the bulk structure, we describe our unit cell by the space group $I \overline{4} 2 d$ (No. 122), which is derived from the inverse tetragonal Heusler structure. This is supported by the observation of a systematic absence of Bragg peaks corresponding to this crystal symmetry (Fig. S1). By modeling the peak intensities,
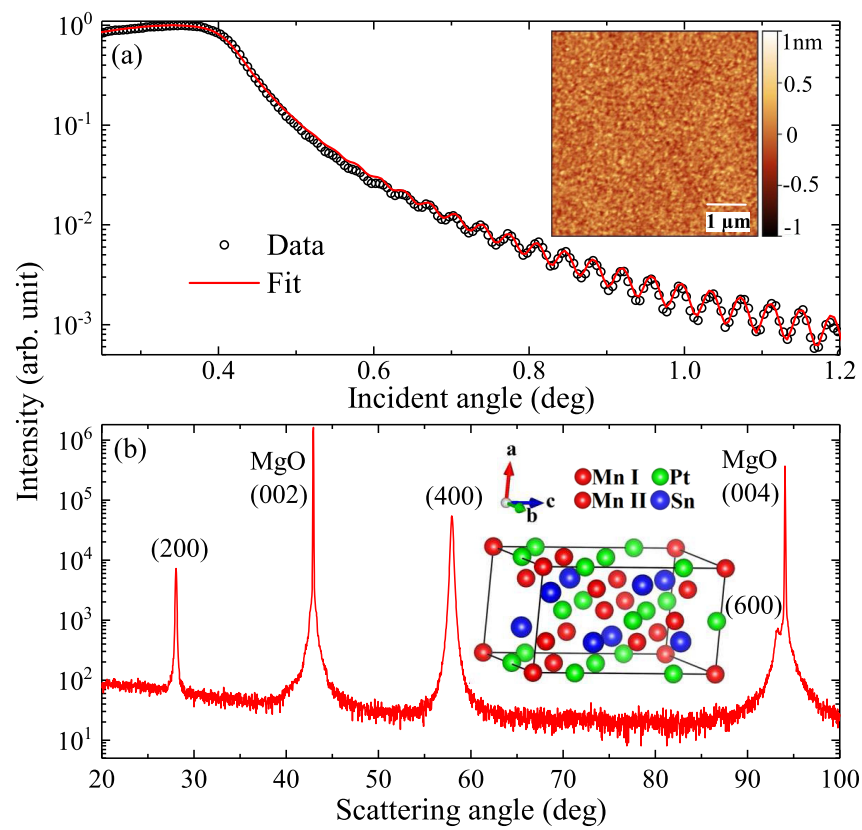

FIG. 1. Structural characterization of the 104-nm-thick $\mathrm{Mn}_{1.5}$ PtSn film. (a) XRR pattern with Kiessig fringes including fit. Inset: AFM image. (b) $\omega-2 \theta$ scans recorded in out-of-plane geometry showing the (200), (400), and (600) peaks as well as the (002) and (004) substrate Bragg peaks. Inset: tetragonal crystal structure. 


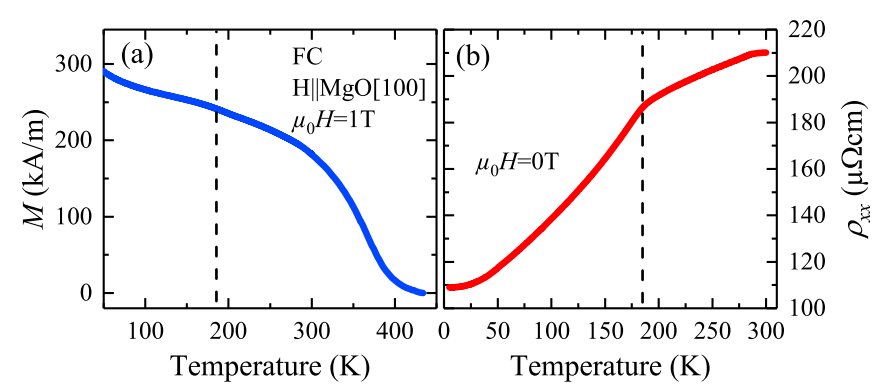

FIG. 2. In-plane magnetization (a) and zero-field resistivity parameters, reflected in the lattice parameters (b) of the $104 \mathrm{~nm}$ thick $\mathrm{Mn}_{1.5} \mathrm{PtSn}$ film as a function of temperature. The dashed lines at $T_{s}=185 \mathrm{~K}$ mark the spin reorientation transition temperature.

we find that Mn atoms occupy the $4 c$ and $8 d(x=0.75)$ positions, while the $\mathrm{Pt}$ and $\mathrm{Sn}$ atoms occupy the $8 c(z=$ $0.23)$ and $8 d(x=0.29)$ positions, respectively. A detailed analysis of the peak positions shows that the film geometry stabilizes the $c$ axis in the film plane, slightly breaking the equivalence of the $a$ and $b$ parameters, reflected in the lattice parameters $a=6.338 \AA \pm 0.004 \AA, b=6.36 \AA \pm 0.01 \AA$ and $c=12.22 \AA \pm 0.03 \AA$.

From the $\{112\}$ pole figure and the comparison of the corresponding azimuthal scan as well as the splitting of highangle peaks (see Figs. S1 and S2 in the Supplemental Material [31]) we conclude that two orientations of the $c$ axis, along [110] and [110] of the $\mathrm{MgO}$ substrate, are present. For the two lattice directions within the film plane, this corresponds to a lattice mismatch of $2.5 \%$ and $6.5 \%$, respectively.

\section{B. Magnetometry and magnetotransport properties}

Figure 2(a) depicts the temperature dependence of $M$ at $1 \mathrm{~T}$ with a single transition at $400 \mathrm{~K}$ representing the Curie temperature for the 104-nm-thick $\mathrm{Mn}_{1.5} \mathrm{PtSn}$ film. A spin reorientation is not clearly evident for this field. Figure 2(b) shows the temperature dependence of $\rho_{x x}$. In analogy to the case of $\mathrm{Mn}_{2} \mathrm{RhSn}$ [28], a change in the slope at $T_{s}=$ $185 \mathrm{~K}$ marks a transition from a collinear $\left(T>T_{s}\right)$ into a noncollinear $\left(T<T_{s}\right)$ magnetic structure following spin reorientation of one Mn sublattice. A similar feature was also observed in related compounds [25-27].

The out-of-plane magnetization for the 104-nm-thick $\mathrm{Mn}_{1.5} \mathrm{PtSn}$ film is shown for 10,150 , and $300 \mathrm{~K}$ at magnetic fields up to $7 \mathrm{~T}$ in Fig. 3(a). The $M(H)$ loops are reminiscent of hard-axis behavior with a small coercive field. We attribute this to the tetragonal $c$ axis lying in the film plane. Here, $M_{S}$ is 415,550 , and $590 \mathrm{kA} / \mathrm{m}$, which correspond to $3.0 \mu_{B} /$ f.u., $4.0 \mu_{B}$ /f.u., and $4.3 \mu_{B} /$ f.u. at 300,150 , and $10 \mathrm{~K}$, respectively, which is comparable to $M_{s}$ determined for the bulk material [27]. The saturation field is estimated to be about $1.2 \mathrm{~T}$ at $300 \mathrm{~K}$, increasing to about $3.5 \mathrm{~T}$ at $10 \mathrm{~K}$.

The magnetoresistance (MR) in Fig. 3(b), recorded with $H$ applied along the out-of-plane direction, is depicted as the ratio $\left[\rho_{x x}(H)-\rho_{x x}(0)\right] / \rho_{x x}(0)$. The MR is negative for all temperatures and is composed of two parts: First, a steep part leveling off around $4 \mathrm{~T}$ and visible at $10 \mathrm{~K}$. This likely originates from the alignment of the spins in the noncoplanar phase and scales with the magnetometry data [Fig. 3(a)].

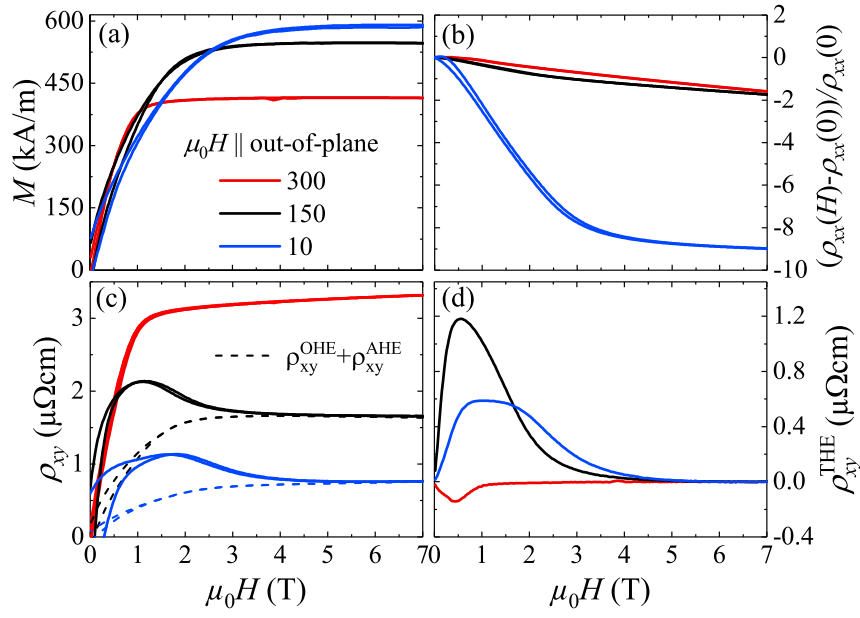

FIG. 3. First quadrant of magnetization curve and resistivities at 10,150 , and $300 \mathrm{~K}$ as a function of applied field in the 104-nm-thick $\mathrm{Mn}_{1.5} \mathrm{PtSn}$ film. (a) Out-of-plane magnetization, (b) magnetoresistance ratio ( $H$ oriented out of plane), (c) Hall resistivity [Eq. (1)] with the dashed lines depicting the ordinary and anomalous Hall contributions, and (d) topological Hall resistivity after subtraction [Eq. (2)] with averaged sweep directions.

Second, a linear field dependent part which does not saturate at $7 \mathrm{~T}$. Furthermore, the absolute value of the MR ratio at $7 \mathrm{~T}$ clearly decreases with increasing temperature.

The Hall resistivity at $300 \mathrm{~K}$ in Fig. 3(c) resembles $M(H)$ [Fig. 3(a)] with a steep increase at low fields and and a linear behavior at high fields. Those two regimes can be attributed to the AHE and the OHE, respectively. Below $T_{s}$, at 150 and $10 \mathrm{~K}$, an additional nonlinear part appears up to approximately $4 \mathrm{~T}$. Here, $\rho_{x y}$ does not trace $M(H)$, which is reminiscent of the THE. The three different contributions can be summarized as

$$
\rho_{x y}=\rho_{x y}^{\mathrm{OHE}}+\rho_{x y}^{\mathrm{AHE}}+\rho_{x y}^{\mathrm{THE}},
$$

where $\rho^{\mathrm{OHE}}$ corresponds to the OHE scaling linearly with applied field $(H), \rho^{\mathrm{AHE}}$ is the AHE scaling with $M$ perpendicular to the film, and $\rho^{\mathrm{THE}}$ represents the THE.

The AHE can arise from intrinsic and/or extrinsic mechanisms, scaling with different powers of the resistivity [2]. Therefore, we write $\rho^{\mathrm{AHE}}=\left(S_{\mathrm{A}} \rho_{x x}^{2}+\alpha \rho_{x x}\right) M$, with $S_{\mathrm{A}}$ corresponding to intrinsic and side-jump scattering and $\alpha$ corresponding to skew scattering. In an independent analysis, we determined from the scaling relation $\rho_{x y} \propto \rho_{x x}^{\beta}$ that the underlying mechanism is of primarily intrinsic origin with $\beta=2.2$ (see Fig. S3 in the Supplemental Material [31]). The zero-field conductivity $\sigma_{x x} \approx 10^{-4} \mathrm{~S} \mathrm{~m}^{-1}$, supports the notion that the intrinsic and side-jump mechanisms dominate [2,32]. Therefore, we focus on the skew scattering independent contributions in our evaluation in the following, taking $\alpha=0$.

In order to quantify the different contributions to the field dependent Hall resistivity, we follow the customarily performed separation process [20]. Therefore, we take into account that only the AHE and OHE contribute to the Hall resistivity once the magnetization is saturated at high fields. Hence, $R_{0}$ and $S_{\mathrm{A}}$ can be obtained through a linear fit to our transport data taken at high magnetic fields, using the 


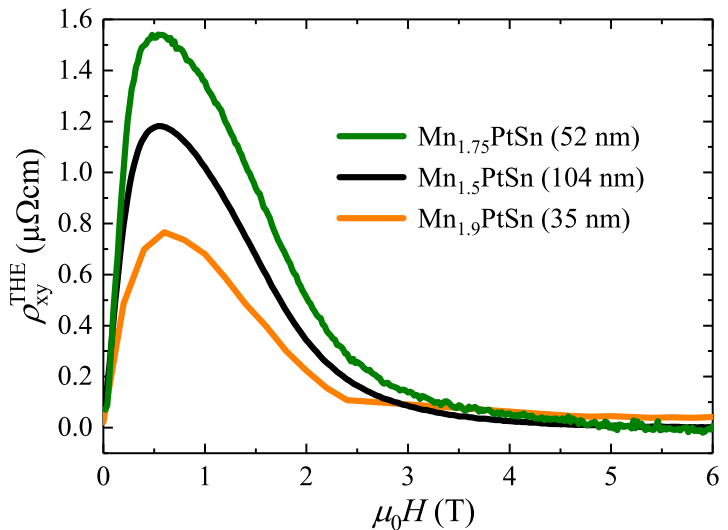

FIG. 4. Topological Hall resistivity (after subtraction, with averaged sweep directions) at $150 \mathrm{~K}$ as a function of applied field for $\mathrm{Mn}_{1.5} \mathrm{PtSn}(104 \mathrm{~nm}), \mathrm{Mn}_{1.75} \mathrm{PtSn}$ (52 nm), and $\mathrm{Mn}_{1.9} \mathrm{PtSn}$ (35 nm).

resistivity $\rho_{x x}$ and the (separately measured) magnetization $M$ as $\rho_{x y} / H=R_{0}+S_{\mathrm{A}} \rho_{x x}^{2} M / H$. Finally, we can calculate the topological Hall resistivity as

$$
\rho_{x y}^{\mathrm{THE}}=\rho_{x y}-R_{0} H+S_{\mathrm{A}} \rho_{x x}^{2} M .
$$

As evident from Fig. 3(d), the THE in the 104-nm-thick $\mathrm{Mn}_{1.5} \mathrm{PtSn}$ film can be observed up to fields of $\mu_{0} H \approx$ $4 \mathrm{~T}$ with a maximum topological Hall resistivity of $\rho_{\max }^{\mathrm{THE}}$ $=1.2 \mu \Omega \mathrm{cm}$ at $150 \mathrm{~K}$. From an analogous analysis in films of $\mathrm{Mn}_{1.75} \mathrm{PtSn}(52 \mathrm{~nm})$ and $\mathrm{Mn}_{1.9} \mathrm{PtSn}(35 \mathrm{~nm})$ at $150 \mathrm{~K}$ we obtained $\rho_{\max }^{\mathrm{THE}}=1.55 \mu \Omega \mathrm{cm}$ and $\rho_{\max }^{\mathrm{THE}}=0.76 \mu \Omega \mathrm{cm}$ with a $T_{s}$ of 187 and $190 \mathrm{~K}$, respectively (Fig. 4). Our data shows that a large THE is present in a wide range of stoichiometries, underlining the robustness of the effect. This is in agreement with the presence of a (weaker) THE, previously reported in bulk $\mathrm{Mn}_{2} \mathrm{PtSn}$ [25] and polycrystalline $\mathrm{Mn}_{2} \mathrm{PtSn}$ films $(I \overline{4} m 2)$ [26]. Notably, in single-crystalline $\mathrm{Mn}_{2} \mathrm{PtSn}$ films $(I \overline{4} m 2)$ [30] with the $c$ axis in the plane, no $T_{s}$ and no THE were observed. We therefore propose that the contradicting observations (presence or absence of the THE in seemingly similar thin films) might be attributed to the different crystal structures and crystal orientations relative to the applied field.

Figure 5 summarizes the temperature evolution of $\rho^{\mathrm{AHE}}$, $\rho_{\max }^{\mathrm{THE}}$, and the field $\mu_{0} H_{\max }^{\mathrm{THE}}$ at which the THE reaches its maximum. $\rho^{\mathrm{AHE}}$ decreases continuously with temperature, having the largest slope around $T_{s}=185 \mathrm{~K}$. The THE appears below $T_{s}$, and thus must be connected with a noncoplanar spin texture at finite fields, with $\rho_{\max }^{\mathrm{THE}}$ peaking at $150 \mathrm{~K}$. Interestingly, $\rho_{\max }^{\mathrm{THE}}$ and $\rho^{\mathrm{AHE}}$ have the same magnitude between 100 and $10 \mathrm{~K}$, suggesting that a similar microscopic mechanism is responsible for both effects. $\mu_{0} H_{\max }^{\mathrm{THE}}$ increases continuously with decreasing temperature following the same trend as the saturation field in the magnetization [Fig. 3(a)].

Since magnetization experiments in films are challenging, the employed extraction procedure is highly susceptible to small misalignments in sample mounting or temperature differences between the transport and magnetometry measurements. This can result in significant errors of the THE values or even mimic non-existent effects. It is therefore unclear whether the THE signature at low fields and above $T_{s}$ [Fig. 3(d)] is genuine or attributable to the THE extraction

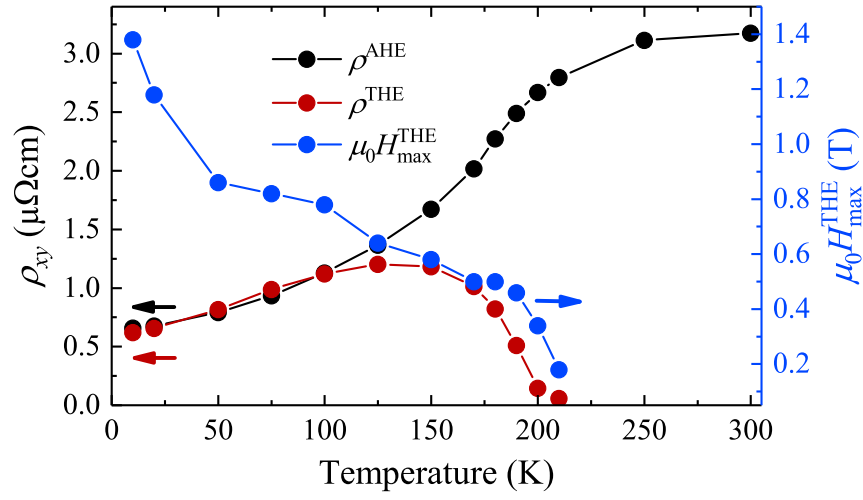

FIG. 5. Anomalous and topological Hall resistivities and field of maximal topological Hall effect as a function of temperature in 104-nm-thick $\mathrm{Mn}_{1.5} \mathrm{PtSn}$ film. The anomalous Hall resistivity was obtained by extrapolating the linear high-field part of the Hall resistivity to $0 \mathrm{~T}$. The maximal topological Hall resistivity is the peak of the nonlinear curvature for each respective temperature at $\mu_{0} H_{\max }^{\mathrm{THE}}$.

process [33]. However, our findings would agree with the presence of antiskyrmions above $T_{s}$ in $\mathrm{Mn}_{1.4} \mathrm{Pt}_{0.9} \mathrm{Pd}_{0.1} \mathrm{Sn}$ [27].

In contrast to the majority of reports on the THE in conjunction with the AHE, we find that in $\mathrm{Mn}_{1.5} \mathrm{PtSn}$ thin films the size of the THE is of the same magnitude as the corresponding AHE. Typically, the AHE by far surpasses the THE $[18,21,24,34]$. Nevertheless, similar behavior as in $\mathrm{Mn}_{1.5} \mathrm{PtSn}$ was also presented in the noncollinear metallic $\mathrm{Mn}_{5} \mathrm{Si}_{3}$ and the correlated oxide charge-transfer insulator $(\mathrm{Ca}, \mathrm{Ce}) \mathrm{MnO}_{3}$ $[17,23]$. Interestingly, one can also find a few examples where the THE appears in conjunction with a vanishing AHE, such as the Weyl semimetal GdPtBi and the helimagnetic metal MnGe $[20,35]$. Thus, the dependence of the underlying mechanism (i.e., skyrmions/bubbles, Weyl points, or noncoplanar magnetic structure) in the respective material system (e.g, thin film, bulk, or multilayer) determines the relation of the THE to the AHE, which can range over several orders of magnitude. The physics regarding the relation of the THE to the AHE have not been completely explored or understood, where in our films we clearly observe a difference in the relation that depends on the spin reorientation transition temperature.

\section{CONCLUSION}

In this work, we report a nontrivial behavior of the Hall response in $\mathrm{Mn}_{1.5} \mathrm{PtSn}$ thin films (space group I $\overline{4} 2 d$ ) identified as the THE. The signature is clearly evident even prior to the customarily performed subtraction of magnetometry data. The THE is present up to a spin reorientation transition temperature, $T_{s}=185 \mathrm{~K}$, and a field of $\mu_{0} H \approx 4 \mathrm{~T}$. The same magnitude of $\rho_{\max }^{\mathrm{THE}}$ and $\rho^{\mathrm{AHE}}$ below $100 \mathrm{~K}$ implies a similar microscopic mechanism for the AHE and THE. While we focused on a 104-nm-thick $\mathrm{Mn}_{1.5} \mathrm{PtSn}$ film, similar experiments in different $\mathrm{Mn}_{2-x} \mathrm{PtSn}$ films show that the THE is robust over various stoichiometries and thicknesses, reaching up to $\rho_{\max }^{\mathrm{THE}}=1.55 \mu \Omega \mathrm{cm}$ at $150 \mathrm{~K}$. All together, $\mathrm{Mn}_{2-x} \mathrm{PtSn}$ is an interesting compound for the understanding and application of transport phenomena in topological magnetic structures. 


\section{ACKNOWLEDGMENTS}

The authors acknowledge funding by the Deutsche Forschungsgemeinschaft (DFG, German Research Foundation) under SPP 2137 (Project No. 403502666), ERC
Advanced Grant No. 742068 "TOPMAT," and EU FET Open RIA Grant No. 766566 (ASPIN). P.S. acknowledges financial support by the International Max Planck Research School for Chemistry and Physics of Quantum Materials (IMPRSCPQM).
[1] A. Fert, V. Cros, and J. Sampaio, Nat. Nanotechnol. 8, 152 (2013).

[2] N. Nagaosa, J. Sinova, S. Onoda, A. H. MacDonald, and N. P. Ong, Rev. Mod. Phys. 82, 1539 (2010).

[3] E. H. Hall, Am. J. Math. 2, 287 (1879).

[4] R. Karplus and J. M. Luttinger, Phys. Rev. 95, 1154 (1954).

[5] J. Smit, Physica 24, 39 (1958).

[6] L. Berger, Phys. Rev. B 2, 4559 (1970).

[7] Z. Fang, N. Nagaosa, K. S. Takahashi, A. Asamitsu, R. Mathieu, T. Ogasawara, H. Yamada, M. Kawasaki, Y. Tokura, and K. Terakura, Science 302, 92 (2003).

[8] J. Ye, Y. B. Kim, A. J. Millis, B. I. Shraiman, P. Majumdar, and Z. Tešanović, Phys. Rev. Lett. 83, 3737 (1999).

[9] P. Bruno, V. K. Dugaev, and M. Taillefumier, Phys. Rev. Lett. 93, 096806 (2004).

[10] Y. Taguchi, Y. Oohara, H. Yoshizawa, N. Nagaosa, and Y. Tokura, Science 291, 2573 (2001).

[11] J. Kübler and C. Felser, Europhys. Lett. 108, 67001 (2014).

[12] K. Manna, Y. Sun, L. Muechler, J. Kübler, and C. Felser, Nat. Rev. Mater. 3, 244 (2018).

[13] L. Wollmann, A. K. Nayak, S. S. P. Parkin, and C. Felser, Annu. Rev. Mater. Res. 47, 247 (2017).

[14] U. K. Rößler, A. N. Bogdanov, and C. Pfleiderer, Nature (London) 442, 797 (2006).

[15] I. Dzyaloshinsky, J. Phys. Chem. Solids 4, 241 (1958).

[16] T. Moriya, Phys. Rev. 120, 91 (1960).

[17] C. Sürgers, G. Fischer, P. Winkel, and H. Löhneysen, Nat. Commun. 5, 3400 (2014).

[18] A. Neubauer, C. Pfleiderer, B. Binz, A. Rosch, R. Ritz, P. G. Niklowitz, and P. Böni, Phys. Rev. Lett. 102, 186602 (2009).

[19] M. V. Berry, Proc. R. Soc. London, Ser. A 392, 45 (1984).

[20] N. Kanazawa, Y. Onose, T. Arima, D. Okuyama, K. Ohoyama, S. Wakimoto, K. Kakurai, S. Ishiwata, and Y. Tokura, Phys. Rev. Lett. 106, 156603 (2011).

[21] S. X. Huang and C. L. Chien, Phys. Rev. Lett. 108, 267201 (2012).

[22] M. Nakamura, D. Morikawa, X. Yu, F. Kagawa, T. Arima, Y. Tokura, and M. Kawasaki, J. Phys. Soc. Jpn. 87, 074704 (2018).
[23] L. Vistoli, W. Wang, A. Sander, Q. Zhu, B. Casals, R. Cichelero, A. Barthélémy, S. Fusil, G. Herranz, S. Valencia, R. Abrudan, E. Weschke, K. Nakazawa, H. Kohno, J. Santamaria, W. Wu, V. Garcia, and M. Bibes, Nat. Phys. (2018), doi: 10.1038/s41567018-0307-5.

[24] K. G. Rana, O. Meshcheriakova, J. Kübler, B. Ernst, J. Karel, R. Hillebrand, E. Pippel, P. Werner, A. K. Nayak, C. Felser, and S. S. P. Parkin, New J. Phys. 18, 085007 (2016).

[25] Z. H. Liu, A. Burigu, Y. J. Zhang, H. M. Jafri, X. Q. Ma, E. Liu, W. H. Wang, and G. H. Wu, Scr. Mater. 143, 122 (2018).

[26] Y. Li, B. Ding, X. Wang, H. Zhang, W. Wang, and Z. Liu, Appl. Phys. Lett. 113, 062406 (2018).

[27] A. K. Nayak, V. Kumar, T. Ma, P. Werner, E. Pippel, R. Sahoo, F. Damay, U. K. Rößler, C. Felser, and S. S. P. Parkin, Nature (London) 548, 561 (2017).

[28] O. Meshcheriakova, S. Chadov, A. K. Nayak, U. K. Rößler, J. Kübler, G. André, A. A. Tsirlin, J. Kiss, S. Hausdorf, A. Kalache, W. Schnelle, M. Nicklas, and C. Felser, Phys. Rev. Lett. 113, 087203 (2014).

[29] A. B. Butenko, A. A. Leonov, U. K. Rößler, and A. N. Bogdanov, Phys. Rev. B 82, 052403 (2010).

[30] Y. Jin, S. Valloppilly, P. Kharel, J. Waybright, P. Lukashev, X. Z. Li, and D. J. Sellmyer, J. Appl. Phys. 124, 103903 (2018).

[31] See Supplemental Material at http://link.aps.org/supplemental/ 10.1103/PhysRevMaterials.3.013001 for additional x-ray diffraction radial scans, pole figure, $\phi$-scan, and power-law scaling of $\mathrm{Mn}_{1.5} \mathrm{PtSn}$.

[32] T. Miyasato, N. Abe, T. Fujii, A. Asamitsu, S. Onoda, Y. Onose, N. Nagaosa, and Y. Tokura, Phys. Rev. Lett. 99, 086602 (2007).

[33] H. Ishizuka and N. Nagaosa, Sci. Adv. 4, eaap9962 (2018).

[34] C. S. Spencer, J. Gayles, N. A. Porter, S. Sugimoto, Z. Aslam, C. J. Kinane, T. R. Charlton, F. Freimuth, S. Chadov, S. Langridge, J. Sinova, C. Felser, S. Blügel, Y. Mokrousov, and C. H. Marrows, Phys. Rev. B 97, 214406 (2018).

[35] T. Suzuki, R. Chisnell, A. Devarakonda, Y. T. Liu, W. Feng, D. Xiao, J. W. Lynn, and J. G. Checkelsky, Nat. Phys. 12, 1119 (2016). 